\title{
Validación de la Escala de Autoeficacia para el Afrontamiento del Estrés en estudiantes universitarios ${ }^{1}$
}

\author{
Carolina Iris Pereyra Girardi², Natalia Páez Vargas ${ }^{3}$, \\ Carla del Valle Ronchieri Pardo ${ }^{4}$, Daniela Agustina Trueba ${ }^{5}$ \\ Universidad del Salvador-Argentina
}

El presente trabajo tiene por objetivos examinar las evidencias de validez de contenido y aparente, analizar evidencias de validez de constructo, estudiar la consistencia interna de las dimensiones que integran el instrumento e investigar evidencias de validez concurrente de la Escala de Autoeficacia para el Afrontamiento del Estrés en estudiantes universitarios. La muestra se conformó por 333 alumnos de una universidad privada argentina. Para esto, se realizó procedimientos de juicio experto y una prueba piloto. Posteriormente se efectuó un estudio factorial confirmatorio comparando dos modelos factoriales de primer orden y de segundo orden. Los resultados evidenciaron un adecuado ajuste del modelo de primer orden, registrando una adecuada consistencia interna y evidencias de validez concurrente. Estos resultados fueron discutidos utilizando las dos concepciones dominantes sobre la autoeficacia.

Palabras clave: autoeficacia, estudio factorial confirmatorio, estudiantes universitarios, validez, confiabilidad.

1 Agradecimientos: Los autores reconocen y agradecen la colaboración de Analía Rivas y Julián Mur, integrantes del proyecto mayor: Autoeficacia para el Afrontamiento del Estrés y la Ansiedad Rasgo-Estado en Estudiantes Universitarios. VRID 1569. Instituto de Investigación en Psicología y Psicopedagogía. El presente trabajo se llevó adelante con financiamiento de la Universidad del Salvador, Facultad de Psicología y Psicopedagogía.

2 Doctora en Humanidades Médicas. Investigadora principal, Instituto de Investigación en Psicología y Psicopedagogía, Universidad del Salvador. Profesora titular de Procesos Básicos IV, Universidad del Salvador. Dirección postal: Marcelo T. de Alvear 1335 - $1^{\circ}$ piso - IIPUS. Contacto: carolina.pereyra@usal.edu.ar ORCID: 0001-7905-5124

3 Licenciada en Psicología. Investigadora adjunta Instituto de Investigación en Psicología y Psicopedagogía Universidad del Salvador. Profesora adjunta de Procesos Básicos III, Universidad del Salvador. Dirección postal: Marcelo T. de Alvear 1335 - $1^{\circ}$ piso - IIPUS. Contacto: nataliapaezv@gmail.com ORCID: 0003-4485-9178

4 Licenciada en Psicología. Investigadora asistente, Instituto de Investigación en Psicología y Psicopedagogía. Dirección postal: Marcelo T. de Alvear 1335 - $1^{\circ}$ piso - IIPUS. Contacto:c. ronchieri@gmail.com ORCID:0001-6251-7329

5 Estudiante de Psicología en la Universidad del Salvador. Practicante del Proyecto de Investigación Autoeficacia para el Afrontamiento del Estrés y la Ansiedad Rasgo-Estado en Estudiantes Universitarios. VRID 1569. Instituto de Investigación en Psicología y Psicopedagogía. Dirección postal: Marcelo T. de Alvear 1335 - $1^{\circ}$ piso - IIPUS. Contacto: truebadaniela@hotmail.com ORCID:0001-6736-0973 


\section{Validation of the Coping with Stress Self-Efficacy Scale in university students}

The aim of this study is to examine evidence of content and face validity, construct validity, the internal consistency of the dimensions that compose the instrument, and concurrent validity. The sample included 333 students from a private Argentine university. Content and face validity was carried out by expert judges and a pilot test. Subsequently, a confirmatory factor analysis was carried out, comparing two factor models of first and second order. The results showed a proper adjustment of the first-order factor model, adequate internal consistency and evidence of concurrent validity. These results are discussed using the two dominant conceptions of self-efficacy.

Keywords: self-efficacy, confirmatory factor analysis, university students, validity, reliability .

Validaçáo da escala de autoeficácia para enfrentar o estresse em estudantes universitarios O presente trabalho tem como objetivos: examinar evidências de validade de conteúdo e aparente, analisar evidências de validade de construto, estudar a consistência interna das dimensóes que compóem o instrumento e investigar evidências de validade concorrente. A amostra incluiu 333 estudantes de uma universidade privada argentina. O estudo de validade de conteúdo e aparente foi realizado através do julgamento de especialistas e um teste piloto. Posteriormente, foi realizado um estudo fatorial confirmatório comparando dois modelos fatoriais de primeira e segunda ordem. Os resultados evidenciaram um ajuste adequado do modelo de primeira ordem, registrando uma consistência interna adequada e evidências de validade concorrente. Esses resultados foram discutidos usando as duas concepçóes dominantes de autoeficácia.

Palavras-chave: autoeficácia, estudo fatorial confirmatório, estudantes universitários, validade, confiabilidade.

\section{Validation de l'échelle d'auto-efficacité pour faire face au stress chez les étudiants universitaires}

Le présent travail vise à examiner les preuves de validité de contenu et apparentes, à analyser les preuves de validité de construction, à étudier la cohérence interne des dimensions composant l'instrument et à rechercher les preuves de validité concurrente de l'échelle d'auto-efficacité pour faire face au stress chez les étudiants universitaires. L'échantillon était composé de 333 étudiants d'une université privée argentine. Pour cela, des procédures de jugement d'experts et un essai pilote ont été effectués. Par la suite, une étude factorielle de confirmation a été réalisée comparant deux modèles factoriels de premier et second ordre. Les résultats ont mis en évidence un ajustement adéquat du modèle de premier ordre, en enregistrant une cohérence interne adéquate et des preuves de validité concurrente. Ces résultats ont été discutés en utilisant les deux conceptions dominantes de l'auto-efficacité. Mots-clés: auto-efficacité, étude factorielle confirmatoire, étudiants universitaires, validité, fiabilité. 
Actualmente, la autoeficacia (AE), constructo introducido por Bandura en 1977 como base de la Teoría Social Cognitiva, se ha posicionado como uno de los más estudiados por la psicología dado su carácter predictor de conducta y su versatilidad de aplicación en diversas áreas de estudio. La Teoría Social Cognitiva propone que los diversos procedimientos psicológicos tienen relación directa con la creación, o bien, el fortalecimiento de las expectativas (Bandura, 1977). Los sujetos generan y desarrollan autopercepciones acerca de su propia capacidad que funcionan como mediadoras para el logro de metas y la toma de decisiones (Ornelas, Blanco, Gastélum y Chávez, 2012; Gutiérrez, Escartí y Pascual, 2011; Sansinenea et al., 2008). De esta manera, surge el concepto de $\mathrm{AE}$, definida como el conjunto de creencias acerca de la efectividad de la conducta para manejar las demandas y desafíos a enfrentar, sobre la base, principalmente, de dos tipos de expectativas: de eficacia y de resultado. Las primeras se refieren al juicio sobre la capacidad personal para realizar acciones efectivas para afrontar determinada situación; las de resultado son las creencias sobre el resultado deseado o esperado que se obtendrá al ejecutar dichas acciones (Bandura, Caprara, Barbaranelli, Gerbino \& Pastorelli, 2003).

Diversos autores sostienen que la percepción que tienen las personas acerca de su propia eficacia es una variable fundamental en la competencia humana debido a que influye en las estrategias, la motivación y la persistencia relacionadas al logro de un objetivo. Del mismo modo, repercute en la respuesta emocional ante situaciones complejas (Blanco et al., 2011; Álvarez et al., 2014; Prieto, 2001; Borzone Valdebenito, 2017; Flores León et al., 2010; Qualter et al., 2015; Rivera Heredia et al., 2016). Adicionalmente, la AE se relaciona con diversos fenómenos mentales y sociales, destacándose su efecto modulador en los procesos cognitivos, emocionales y conductuales. La riqueza del constructo radica en el vínculo con otras variables psicosociales 
de importancia tales como el afrontamiento, la ansiedad y la motivación (Pereyra Girardi et al., 2016).

En concordancia con los postulados de Bandura (1977, 1986), Smith (1989) señala que las creencias de AE no son características personales únicas e invariables, sino que cambian influenciadas por tres dimensiones: magnitud, fuerza y generalidad. Estas dimensiones definen si un evento o situación es o no estresante para una persona en particular. La magnitud de la $\mathrm{AE}$ se refiere al número de pasos de dificultad creciente que la persona cree que es capaz de superar; la fuerza de la $\mathrm{AE}$ consiste en cuán convencida se encuentra de poder llevar adelante dicha conducta y, finalmente, la generalidad de la $\mathrm{AE}$ es la medida en la que las experiencias de éxito y fracaso pueden extenderse a otras conductas o contextos similares (Smith, 1989).

Existen dos concepciones acerca de la AE. Algunos autores la consideran un rasgo global que actúa de modo general en diversos aspectos de las personas, influenciando así sus creencias, acciones y emociones (Luszczynska, Gibbons, Piko \& Tekozel, 2004; Luszczynska, Scholz \& Schwarzer, 2005; Schwarzer \& Jerusalem, 1995). Para esta concepción, la $\mathrm{AE}$ podría explicar un amplio espectro de conductas sobre cuán efectiva puede ser una persona en una variedad de situaciones demandantes a enfrentar (Luszczynska et al., 2004). Sin embargo, el mismo Bandura (2006) señala que la AE debe conceptualizarse de una manera específica, circunscripta a determinados ámbitos de funcionamiento del sujeto. Siguiendo esta segunda conceptualización de AE específica de dominio, la relevancia del constructo ha sido comprobada en diversos campos de estudio: autoeficacia académica, autoeficacia deportiva o autoeficacia social, entre otros posibles (Blanco, Aguirre, Barrón y Blanco, 2016; Peinado, Ornelas, Blanco y González, 2015; Reigal Garrido et al., 2013; Rivera Heredia et al., 2016).

Teniendo en cuenta la especificidad de dominio de la $\mathrm{AE}$, se comenzó a estudiar aquellas creencias personales involucradas en el afrontamiento del estrés, y sus implicaciones en las áreas de funcionamiento humano, su relación con la salud y el bienestar, formulando el concepto de Autoeficacia Específica para el Afrontamiento del Estrés 
—AEAE - (Bandura, 1997; Godoy-Izquierdo et al., 2004a; GodoyIzquierdo et al., 2004b) definida por Godoy-Izquierdo (2004a) como "el conjunto de creencias en los recursos personales para manejar las situaciones demandantes y estresantes de una forma eficaz y competente, esto es, para reducir, eliminar o incluso prevenir el estrés experimentado, para disminuir su impacto y para controlar, de esta forma, sus consecuencias no deseadas" (pp.155-156).

A este respecto, es importante señalar que Bandura (2001) considera necesario diferenciar la autoeficacia percibida de otros constructos tales como autoestima, locus de control y expectativas de resultados, ya que pueden ser tratados como sinónimos al estar relacionados con el afrontamiento del estrés. La eficacia percibida es un juicio sobre la capacidad para enfrentar la situación amenazante; la autoestima, por su parte, es un juicio de valoración personal, no solo ante la situación desafiante, sino que implica una evaluación más amplia que hace el individuo de sí mismo y su conducta. El locus de control no se relaciona con la capacidad percibida, sino con creencias acerca de las eventualidades o consecuencias de resultados determinados por acciones propias o por agentes externos.

Para el caso de la AEAE, Godoy-Izquierdo et al. (2004a) proponen dos tipos de expectativas posibles: las expectativas de eficacia y las de resultado. Las expectativas de eficacia refieren a los juicios subjetivos sobre las propias capacidades, tanto para organizar como para ejecutar eficazmente acciones y regular emociones. Por otra parte, las expectativas de resultado son las creencias personales de que ciertas acciones conducirán a los resultados deseados. En este sentido, las personas que poseen un alto nivel de autoeficacia confían en su capacidad para manejar adecuadamente las demandas ambientales y tienden a percibir las situaciones estresantes como un reto y no como una amenaza incontrolable (Bandura, 1997, 2001). La AE no sólo puede amortiguar las exigencias ambientales minimizando el impacto de sus efectos negativos, sino que incluso puede aportar bienestar cuando se responde de manera adecuada a los requerimientos ambientales (Grau, Salanova y Peiró, 2000; Salanova, Grau, Llorens y Schaufelli, 2001). 
Estudios recientes han evidenciado el rol mediador de la autoeficacia en población universitaria señalando, asimismo, que contribuye a una mejor salud mental y física (Chau y Vilela, 2017).

El presente trabajo busca validar la Escala de Autoeficacia para el Afrontamiento del Estrés (EAEAE) en estudiantes universitarios residentes en la Ciudad Autónoma de Buenos Aires. Se trata de una escala breve diseñada para evaluar ambos constructos (Autoeficacia - Afrontamiento del estrés) por su mutua influencia en el manejo de situaciones nuevas, demandantes y decisivas (Godoy-Izquierdo et al., 2008). Para la validación, se plantean cuatro objetivos: 1) Examinar evidencias de validez de contenido y aparente, 2) Analizar evidencias de validez de constructo, 3) Estudiar la consistencia interna de las dimensiones que integran el instrumento, 4) Investigar evidencias de validez concurrente.

\section{Método}

\section{Participantes}

Validez de contenido y estudio piloto

Fueron convocados para la valoración del contenido de los reactivos cinco jueces expertos en psicología cognitiva, en función a su alto conocimiento en la temática (Escobar-Pérez y Cuervo-Martínez, 2008). Por otra parte, 15 estudiantes de una universidad privada argentina participaron de la prueba piloto.

Evidencias de Validez de Constructo-Análisis Factorial Confirmatorio y Validez Concurrente y Consistencia Interna

Los datos fueron recogidos mediante un muestreo por conveniencia. Se trabajó con estudiantes universitarios de la Ciudad Autónoma de Buenos Aires adoptando como criterios de inclusión que los alumnos fueran regulares al momento de la toma de datos, excluyéndose los casos en que no se hubiera completado la totalidad del protocolo, asegurando así la ausencia de datos perdidos. 
La muestra se conformó por 333 estudiantes (79\% mujeres y $21 \%$ varones), de entre 18 y 35 años $(M=24.82 ; D E=5.33)$, todos ellos pertenecientes a una universidad privada argentina. Los estudiantes se encontraban cursando distintos momentos de la carrera (10\% primer año, $30 \%$ segundo año, $32 \%$ tercer año, $25 \%$ cuarto ańo y $3 \%$ quinto año) y en diferentes bandas horarios (71\% turno noche, $18 \%$ turno mañana y $11 \%$ turno tarde). El $72 \%$ de la muestra trabaja, mientas que el $28 \%$ restante no.

\section{Medición}

Cuestionario de datos generales: Cuestionario, elaborado ad hoc, que recaba información a través de preguntas cerradas con alternativa fija, sobre la muestra, en las variables: edad, sexo, turno que cursa y situación laboral.

Escala de Autoeficacia para el Afrontamiento del Estrés (EAEAE): (Godoy-Izquierdo y Godoy, 2001, 2006; Godoy-Izquierdo., 2004a; Godoy-Izquierdo et al., 2004b; Godoy-Izquierdo et al., 2004c): Instrumento compuesto por 8 ítems, de los cuales 4 (ítems 2, 4, 5, 7) evalúan el componente de expectativa de eficacia (EE) del constructo autoeficacia, y los 4 restantes $(1,3,6,8)$ el de expectativa de resultado (ER). Las respuestas se presentan en formato tipo Likert con 5 alternativas que van desde: "Completamente en desacuerdo" hasta "Completamente de acuerdo", con valores del 1 al 5. Se obtiene también una puntuación total, resultado de la suma de las dos puntuaciones parciales. La escala validada en población espańola cuenta con evidencias de validez de constructo —análisis de componentes principales y validez concurrente-y consistencia interna (Godoy-Izquierdo et al., 2008).

Escala de Autoeficacia General: Se utilizó la adaptación argentina (Brenlla, Aranguren, Rossaro y Vázquez, 2010) de la Escala de Autoeficacia General de Jerusalem \& Schwarzer (1992). El instrumento evalúa el aspecto general de la autoeficacia, es decir, el sentido amplio y estable de competencia personal sobre la percepción de efectividad para afrontar situaciones estresantes. La escala incluye 10 ítems con escalamiento tipo Likert de cuatro puntos, correspondientes a las opciones 
de respuesta: nunca, pocas veces, a veces, y siempre. Las puntuaciones oscilan entre los 10 y 40 puntos. Los valores más altos indican una mejor percepción de la autoeficacia. La consistencia interna de los ítems es apropiada, con valores de alfa de Cronbach de .76.

\section{Procedimiento}

Validez de contenido y prueba piloto

Para realizar la evaluación de contenido de los 8 ítems que componen la Escala de Autoeficacia para el Afrontamiento del Estrés, se convocó un grupo de cinco expertos en Psicología Cognitiva, seleccionados por su experticia y conocimiento (Escobar-Pérez y Cuervo-Martínez, 2008). Cada experto recibió un cuadernillo que contenía la definición teórica del constructo y la descripción de las dimensiones que lo componen —Expectativas de Resultado o Expectativas de Eficacia-. Seguidamente, se presentó un listado con la totalidad de los reactivos de la escala y la consigna de clasificar, según su juicio, a cada uno de los ítems en la dimensión que mejor representa. Se adoptó como criterio para decidir la conservación o eliminación de cada ítem el cálculo del Índice de Acuerdo entre Jueces, que se calculó dividiendo la cantidad de expertos que acordaron la misma clasificación para un ítem, y la totalidad de jueces que participaron — cinco jueces- (Tinsley \& Weiss, 1975). Se conservaron todos los reactivos que hubieran logrado un consenso total de los cinco jueces que participaron de la experiencia. Los ítems resultantes del estudio de contenido fueron sometidos a una prueba piloto con 15 estudiantes, a los cuales se les entrego un protocolo junto con la consigna de leer atentamente las instrucciones y todos los ítems que conforman la escala. Posteriormente, se les entregó una planilla dónde debían consignar si habían comprendido o no las instrucciones y los ítems.

Estudio de Validez de Constructo - Análisis Factorial Confirmatorio y Validez Concurrente- y Consistencia Interna

La recolección de datos fue realizada por tres psicólogas y tres alumnas avanzadas de psicología, todas entrenadas para esta tarea. 
La toma de datos se llevó adelante durante el horario de clases, distribuidas en tres turnos, mañana, tarde y noche. La participación de los estudiantes fue voluntaria y anónima, se les explicó que no recibirían beneficio económico por ella y que podían interrumpir en cualquier momento la prueba sin dar ninguna explicación. Se solicitó, antes de continuar con el protocolo de investigación, la firma del consentimiento informado dónde se explicitaba el objetivo del estudio, la participación voluntaria, el anonimato de sus respuestas y la utilización de los datos con fines científicos.

Inicialmente se llevó a cabo un análisis factorial confirmatorio con el fin de comparar el modelo de segundo orden, propuesto por los autores originales, que posibilita el cálculo de un puntaje total de la técnica (Godoy-Izquierdo et al., 2008), con un modelo bifactorial de primer orden. Los modelos fueron estimados a partir de matrices de correlaciones policóricas por ser las variables en estudio de naturaleza ordinal (Morata-Ramírez \& Holgado-Tello, 2013; Rhemtulla, Brosseau \& Savalei, 2012). Se empleó además el método de estimación AGLS (Arbitrary Distribution Generalized Least Squares), robusto para análisis de un bajo número de reactivos de naturaleza ordinal y con una muestra amplia (Blunch, 2016). Los modelos fueron evaluados a partir de los índices de ajuste NFI (Normed Fit Index), NNFI (Non-Normed Fit Index), CFI (Comparative Fit Index), RMSEA (Root Mean Square Error of Approximation). Por otro lado, la consistencia interna fue estimada a través del coeficiente alfa ordinal dado que este índice resulta el más apropiado para evitar infraestimaciones al trabajar con variables ordinales (Elosua Oliden y Zumbo, 2008).

Luego, a partir del modelo conservado del procedimiento anterior, se continuó con el estudio de validez concurrente correlacionando mediante el coeficiente rho de Spearman las dimensiones del modelo con el puntaje de la escala de autoeficacia general validada localmente (Brenlla et al., 2010), replicando de este modo el estudio de los autores originales. Se utilizó la correlación de Spearman puesto que la muestra no cumplía con el supuesto de normalidad. 


\section{Análisis de datos}

Para estudiar la Validez de Contenido se empleó Índice de Acuerdo entre Jueces para ponderar el contenido de los reactivos en análisis (Tinsley \& Weiss, 1975). Para el análisis factorial confirmatorio y de consistencia interna se empleó el software EQS 6.2, mientras que el análisis de validez concurrente se llevó a cabo mediante el software SPSS 21.

\section{Resultados}

Estudio de Evidencias de Validez de Contenido y Prueba Piloto

Del juicio experto se registró un total consenso entre evaluadores en todos los ítems, — coeficiente de 1-, en cuanto a su clasificación en las dimensiones para las que fueron diseñados originalmente. Con respecto a la prueba piloto, la totalidad de los estudiantes afirmaron comprender correctamente el contenido de los ítems y la consigna, por lo que se decidió no hacer ninguna modificación al instrumento en esta instancia.

\section{Análisis factorial confirmatorio}

Se comparó la estructura bidimensional propuesta con un modelo de segundo orden a fin de valorar cuál de los dos presentaba un mejor ajuste a los datos empíricos locales. La Figura 1 presenta el modelo bidimensional en donde las dos variables latentes se encuentran relacionadas.

La Figura 2 expone un modelo de segundo orden en donde las variables latentes, Expectativa de Eficacia y Expectativa de Resultados, convergen hacia una variable de nivel superior denominada Eficacia Total. 
Validación de la Escala de Autoeficacia para el afrontamiento del estrés en estudiantes / Pereyra et al.

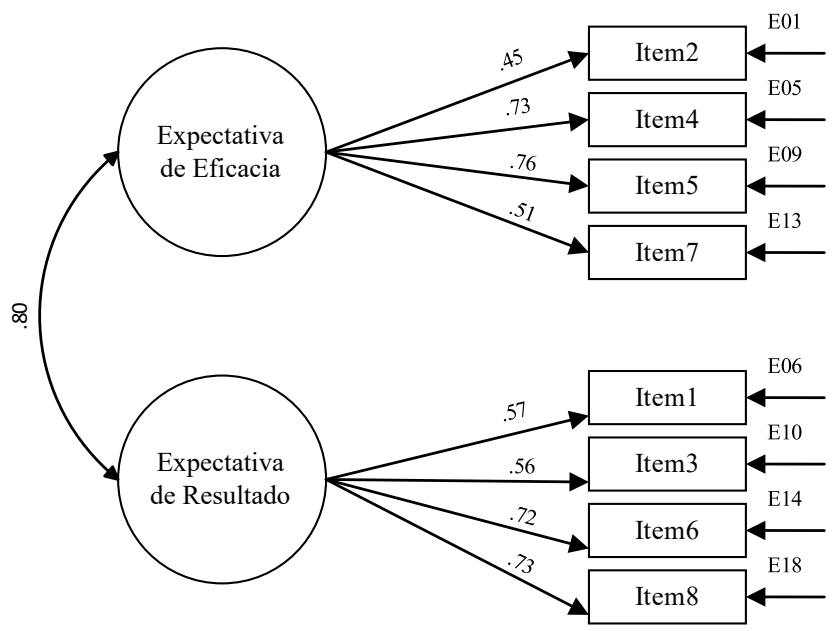

Figura 1. Gráfico de Primer Orden

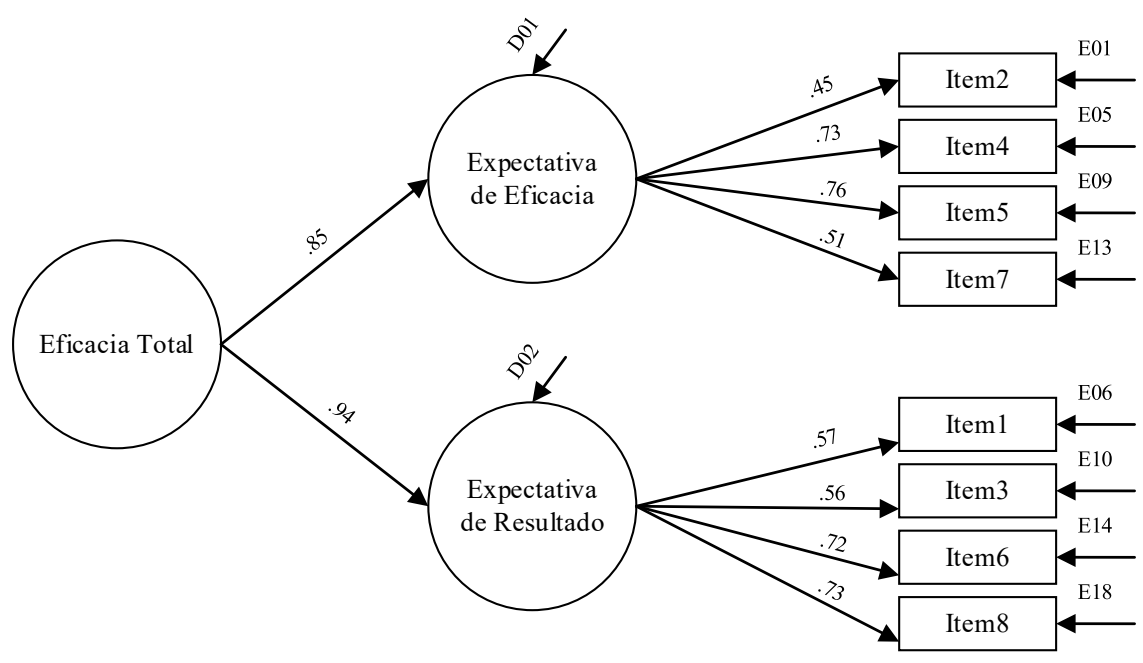

Figura 2. Gráfico de Segundo Orden 
Pasando ahora a la lectura del ajuste de ambos modelos es posible ver que el del primer orden presenta guarismos adecuados en todos índices NFI, NNFI, y CFI ( $>.90)$, mientras que el de segundo orden no alcanza dichos valores. Lo mismo ocurre con el índice RMSEA, registrándose para el modelo de primer orden un valor aceptable (<.08), no así el modelo de segundo orden Dado estos resultados es que resulta pertinente conservar el modelo de primer orden (Tabla 1).

\section{Tabla 1}

Indices de Ajuste

\begin{tabular}{lccccccl}
\hline & \multicolumn{6}{c}{ Índices de Ajuste } \\
\cline { 2 - 8 } Modelo & $\mathrm{z}^{2}$ & $g l$ & NFI & NNFI & CFI & IFI & RMSEA (IC) \\
\hline Primer Orden & 356.297 & 28 & .910 & .924 & .954 & .836 & $.052(.023, .79)$ \\
Segundo Orden & 356.297 & 28 & .792 & .752 & .832 & .836 & $.094(0.71,0.116)$ \\
\hline
\end{tabular}

Nota: Se consideran como factores significativos: los índices NFI, NNFI, y CFI $(>.90)$ y el índice RMSEA (<.08)

\section{Consistencia Interna}

A partir del análisis anterior es que se procedió a estimar el coeficiente alfa ordinal para las dimensiones correspondientes al modelo de primer orden. Se obtuvo un índice de Escala de Expectativa de Eficacia .70, y para la Escala de Expectativa Resultado .74, ambos considerados apropiados, de acuerdo a los criterios usuales (Fleiss, Levin \& Paik, 2004).

\section{Validez concurrente}

Para estudiar evidencias de validez concurrente se procedió a correlacionar los puntajes de las dos dimensiones correspondientes al modelo de primer orden - Expectativa de Eficacia, Expectativa de Resultadoscon el puntaje de la Escala de Autoeficacia General (EAG) de Jerusalem \& Schwarzer (1992) en la adaptación argentina (Brenlla et al., 2010).

Se observan valores de correlaciones significativas de débiles a moderados, entre los puntajes obtenidos en la EAEAE Eficacia Resultado y los obtenidos en la EAG. Las correlaciones, aunque débiles, 
Validación de la Escala de Autoeficacia para el afrontamiento del estrés en estudiantes / Pereyra et al.

indicarían que a mayor expectativa de resultado y de eficacia para el afrontamiento al estrés mayor autoeficacia general. Los resultados coinciden con los encontrados por los autores de la versión original dónde se verificó una correlación débil a moderada y positiva entre la EAEAE Dimensión de Resultado y de Eficacia y AEG (Tabla 2).

\section{Tabla2}

Matriz de correlaciones entre los puntajes en la EAEAE y los puntajes en la $E A G$

\begin{tabular}{lc}
\hline & Escala Autoeficacia General (EAG) \\
\hline Expectativa de Eficacia (EE) & $.529^{* *}$ \\
Expectativa de Resultado (ER) & $.276^{* *}$ \\
\hline
\end{tabular}

Nota: Coeficientes Rho de Spearman ${ }^{* *} \mathrm{p} \leq .01$

\section{Discusión}

Conforme a los análisis efectuados sobre la adaptación argentina de la escala de autoeficacia para el afrontamiento del estrés se asume que la misma cuenta con características técnicas apropiadas, en estudiantes universitarios del área metropolitana de Buenos Aires. Cabe señalar que la versión original EAEAE (Godoy-Izquierdo et al., 2008) fue realizada en población general con un $\mathrm{n}=812$ sujetos adultos $(508$ mujeres y 304 hombres). Se destaca que el presente estudio confirmatorio fue llevado a cabo en población estudiantil. No obstante, la cantidad de sujetos que formaron parte de la muestra resulta adecuada de acuerdo con los estándares de psicometría que sugieren 10 sujetos por ítem (Martínez Arias, 1996).

Se comenzó por llevar adelante estudios de validez de contenido y aparente que permitió conservar la totalidad de los ítems. En cuanto al análisis de contenido, se verificó la apropiada pertenencia de los ítems con relación a las dimensiones propuestas por los autores originales. Se obtuvo un acuerdo total en los jueces expertos participantes en este estudio (Aiken, 1985). Asimismo, de su lectura se desprende que los elementos poseían una importancia diferente entre sí, siendo algunos 
más representativos que otros con respecto a la dimensión a la que respondían. Por otra parte, el estudio de validez aparente, realizado en el estudio piloto, no registró sugerencias consensuadas por parte de los estudiantes, no se recibieron comentarios negativos en cuanto a la claridad y a la pertinencia de la consigna y de los ítems. Respecto a la consistencia interna de la EAEAE, se obtuvieron valores adecuados y similares al estudio previo (Godoy-Izquierdo et al., 2004a).

Con el fin de testear la adecuación de los datos empíricos respecto a los previamente hallados por los autores originales en Espańa, se llevó adelante el análisis factorial confirmatorio. Para ello, se compararon dos estructuras factoriales: una de primer orden y una de segundo orden. Se pudo observar que la estructura de primer orden obtuvo mejores índices de ajuste respecto a la de segundo orden. En tal sentido, hay dos concepciones acerca de la AE, una de ellas apoya un sentido global del constructo: la AE sería, desde esta concepción, una competencia personal, amplia y estable, sobre cuán efectiva puede ser la conducta de una persona para afrontar una variedad de situaciones estresantes (Choi, 2004; Luszczynska, Gibbons, Piko \& Tekozel, 2004; Luszczynska, Scholz \& Schwarzer, 2005; Baessler y Schwarzer, 1996). Sin embargo, otra postura sostiene que hay especificidades de dominio de la AE referidas a la acción, por lo cual ya no sería un rasgo global, unívoco, sino que las autopercepciones de la $\mathrm{AE}$ pueden variar dependiendo del ámbito en el que se actúa, y las expectativas de AE también son diferenciadas dependiendo el ámbito de acción (García-Fernández et al., 2010). Esta definición pone énfasis en la especificidad del rango de acción al que se refiere la $\mathrm{AE}$, ya que no sería un rasgo global; cada persona puede tener un desempeńo diferente dependiendo del ámbito en el que actúa (Escobar y Pérez, 2017).

El propio Bandura (1997), señala que las creencias que posee un individuo sobre sus capacidades para organizar y ejecutar diversas acciones están dirigidas a situaciones y tareas específicas. Esta segunda conceptualización explicaría la diversidad de escalas que evalúan dominios de la AE referidos, por citar algunos, a: Conductas Académicas (Blanco Vega, Martínez Marín, Zueck Enríquez y Gastélum Cuadras, 
2011) a la resolución de problemas y comunicación (Peinado, Ornelas, Blanco y González, 2015), a conducta alimentaria y salud física (Blanco, Ornelas, Viciana y Rodríguez, 2016), a Autoeficacia Profesional (Grau, Martínez, Agut \& Salanova, 2001). En relación con lo anteriormente expuesto, el trabajo reciente de Chau y Vilela (2017), sugiere el estudio de la autoeficacia como específica de dominio y señalan como lineamientos para futuras investigaciones, el estudio de la $\mathrm{AE}$ y el estrés en estudiantes universitarios.

La estructura de primer orden la EAEAE, sugerida en el presente trabajo, sería más afín a la concepción específica de dominio de la $\mathrm{AE}$, una orientada a los resultados y otra a la eficacia, mientras que, en relación con el criterio de validez externa utilizado, se hallaron correlaciones significativas y moderadas similares a la de los autores originales, aunque levemente inferiores. Si bien las correlaciones resultan bajas, las mismas son valores esperables y aceptables para las ciencias sociales (Hemphill, 2003).

Respecto a las limitaciones del estudio, se señala que el mismo fue realizado en población universitaria, con alumnos regulares de las carreras de psicología y psicopedagogía, en su mayoría mujeres (79\%), residentes de la Ciudad Autónoma de Buenos Aires. Se sugiere replicar el estudio en una muestra más grande y heterogénea que incluya, por ejemplo, alumnos de otras carreras, de universidades públicas, con una paridad de género mayor y que sean residentes de otras regiones del país. Asimismo, serían deseables estudios mayores, para replicar el trabajo en población general, subrayando las diversas áreas de aplicación que el constructo posee.

Cabe destacar que la EAEAE conserva adecuadas propiedades de fiabilidad, consistencia interna, validez de constructo y validez concurrente. Estos datos permiten disponer de un instrumento breve, de fácil administración, apropiado para la evaluación e investigación sobre la autoeficacia específica para el afrontamiento del estrés a nivel local. Respecto a los ámbitos de aplicación, por las características del instrumento y la versatilidad de aplicación del concepto autoeficacia, permite contar con una herramienta útil en contextos educativos, laborales, de salud y de investigación. La escala propuesta conserva la versión de los autores originales, evidenciándose como una medida precisa, fiable y válida. 


\section{Referencias}

Aiken, L. (1985). Psychological testing and assessment. Boston: Allyn and Bacon, Inc.

Álvarez, P., Santiviago, C., López, D., Dare, R. \& Rubio, V. (2014). Competencias de adaptabilidad y expectativas del alumnado en proceso de transición a la Educación Superior: un estudio transnacional en España, Uruguay e Italia. IV Conferencia Latinoamericana sobre el Abandono en la Educación Superior. Universidad de Antioquía. Antioquía (Colombia).

Baessler, J. y Schwarzer, R. (1996). Evaluación de la autoeficacia: Adaptación española de la escala de Autoeficacia General. Ansiedady Estrés, 2, 1-8.

Bandura A. (2006) Guide for constructing self-efficacy scales. En F. Pajares, T.C. Urdan (Eds.), Self-efficacy beliefs of adolescents (pp. 30-37). Greenwich: Age Publishing, Inc.

Bandura, A. (1977). Self-Efficacy: Toward a unifying theory of behavioral change. Psychological Review, 84 (2), 191-215. https://doi. org/10.1037//0033-295x.84.2.191

Bandura, A. (1986). Social foundations of thought and action: A social cognitive theory. Englewood Cliffs, NJ: Prentice-Hall.

Bandura, A. (1997). Self-efficacy: The exercise of control. New York: Freeman.

Bandura, A. (2001). Social cognitive theory: an agentic perspective. Annual Review of Psychology, 52, 1-26. https://doi. org/10.1111/1467-839x.00024

Bandura, A., Caprara, G.V., Barbaranelli, C., Gerbino, M. \& Pastorelli, C. (2003). Role of affective self-regulatory efficacy in diverse spheres of psychosocial functioning. Child Development, 74, 769-782. https://doi.org/10.1111/1467-8624.00567

Blanco Vega, H., Martínez Marín, M., Zueck Enríquez, M. y Gastélum Cuadras, G. (2011). Análisis psicométrico de la escala autoeficacia en conductas académicas en universitarios de primer ingreso. Revista Electrónica Actualidades Investigativas en Educación, 11(3), 1-27. 
Blanco, H., Aguirre, J.F., Barrón, J.C. y Blanco, J.R. (2016). Composición factorial de la Escala de Autoeficacia Académica en universitarios mexicanos. Formación Universitaria, 9(2), 81-88. http://dx.doi.org/10.4067/S0718-50062016000200009

Blanco, H., Martínez, A., Ornelas, M. Flores, F. \& Peinado, J. (2011). Validación de las escalas de autoeficacia en conductas académicas y cuidado de la salud. México: Doble Hélice.

Blanco, J.R., Ornelas, M., Viciana, J. y Rodríguez, J.M. (2016). Composición factorial de una escala de autoeficacia en el cuidado de la alimentación y salud física en universitarios mexicanos. Nutrición Hospitalaria, 33(2), 379-385. https://dx.doi.org/10.20960/ nh. 120

Blunch, N. (2016). Introduction to structural equation modeling using IBM SPSS statistics and EQS. London: Sage.

Borzone Valdebenito, M. (2017). Autoeficacia y vivencias académicas en estudiantes universitarios. Acta Colombiana de Psicología, 20(1), 266-274. https://doi.org/10.14718/acp.2017.20.1.13

Brenlla M. E., Aranguren M., Rossaro M. F. y Vázquez N. (2010). Adaptación para Buenos Aires de la Escala de Autoeficacia General. Interdisciplinaria, 27 (1), 77-94.

Chau, C., Vilela, P. (2017). Determinantes de la salud mental en estudiantes universitarios de Lima y Huánuco. Revista de Psicología, 35(2), 387-422. https://dx.doi.org/10.18800/psico.201702.001

Choi, N. (2004). Sex role group differences in specific, academic, and general self-efficacy. The Journal of Psychology, 138, 149-159. https://doi.org/10.3200/jrlp.138.2.149-159

Elosua Oliden, P. y Zumbo, B.D. (2008) Coeficientes de fiabilidad para escalas de respuesta categórica ordenada. Psicothema, 20(4), 896901. Recuperado de http://www.psicothema.com/pdf/3572.pdf

Escobar G.A. y Pérez V. C. (2017). Estructura factorial y confiabilidad de una escala de autoeficacia académica en estudiantes chilenos de Fonoaudiología. Educación Médica Superior, 31(3). Recuperado de http://ems.sld.cu/index.php/ems/article/view/751/554 
Escobar-Pérez, J. y Cuervo-Martínez, A. (2008). Validez de contenido y juicio de expertos: una aproximación a su utilización. Avances en Medición, 6, 27-36. Recuperado dehttp://www.humanas.unal. edu.co/psicometria/files/7113/8574/5708/Articulo3_Juicio_ de_expertos_27-36.pdf

Fleiss, J. L., Levin B. \& Paik, M. C. (2004). Statistical methods for rates and proportions. New York: Wiley.

Flores León, A., González-Celis Rangel, A. L. y Valencia Ortiz, A. (2010). Validación del Instrumento de Autoeficacia para Realizar Conductas Saludables en niños mexicanos sanos. Psicología y Salud, 20(1), 23-30. Recuperado de http://www.uv.mx/psicysalud/psicysalud-20-1/20-1/araceli-flores-leon.pdf

García-Fernández, J. M., Inglés, C., Torregrosa, M. S., Ruiz-Esteban, C., Díaz-Herrero, Pérez-Fernández, E. y Martínez-Monteagudo, M. C. (2010). Propiedades psicométricas de la Escala de Autoeficacia Percibida Específica de Situaciones Académicas en una muestra de estudiantes españoles de Educación Secundaria Obligatoria. European Journal of Education and Psychology, 3(1), 61-74. https://doi.org/10.30552/ejep.v3i1.51

Godoy, J.F. y Godoy-Izquierdo, D. (2003). Escala de Balance Afectivo. Versión experimental no publicada.

Godoy-Izquierdo, D. y Godoy, J.F. (2001). Escala de Autoeficacia Específica para el Afrontamiento del Estrés (EAEAE). Versión experimental no publicada.

Godoy-Izquierdo, D., Godoy García, J.F., López-Chicheri García, I., Martínez Delgado, A., Gutiérrez Jiménez, S. y Vázquez Vázquez, L. (2008). Propiedades psicométricas de la escala de autoeficacia para el afrontamiento del estrés (EAEAE). Psicothema, 20(1), 155-165. Godoy-Izquierdo, D., Godoy, J.F., López, F., Garrido, E., Valor, I., Vélez, M. y León, R. (2004b). Propiedades psicométricas de la «Escala de Autoeficacia Específica para el Afrontamiento del Estrés» II: Validez factorial. $2^{\circ}$ Congreso Hispano-Portugués de Psicología, Lisboa (Portugal). 
Godoy-Izquierdo, D., Godoy, J.F., López, F., Valor, I., Vélez, M., Garrido, E. y León, R. (2004c). Propiedades psicométricas de la «Escala de Autoeficacia Específica para el Afrontamiento del Estrés» III: Validez convergente. $2^{\circ}$ Congreso Hispano-Portugués de Psicología, Lisboa (Portugal).

Godoy-Izquierdo, D., Godoy, J.F., López, F., Vélez, M., Valor, I., Garrido, E. y León, R. (2004a). Propiedades psicométricas de la «Escala de Autoeficacia Específica para el Afrontamiento del Estrés» I: Fiabilidad. $2^{\circ}$ Congreso Hispano-Portugués de Psicología, Lisboa (Portugal).

Godoy-Izquierdo, D. y Godoy, J.F. (2006). Escala de Autoeficacia Específica para el Afrontamiento del Estrés (EAEAE). En V.E. Caballo (Dir.), Manual para la evaluación clínica de los trastornos psicológicos. Madrid: Pirámide.

Grau, R., Martínez, I., Agut, S. \& Salanova, M. (2001). Safety attitudes and their relationship to safety training and generalized self-efficacy. International Journal of Occupational Safety and Ergonomics, 8, 23-35.

Grau, R., Salanova, M. y Peiró, J.M. (2000). Efectos moduladores de la autoeficacia en el estrés laboral. Apuntes de Psicología, 18(1), 57-75.

Gutiérrez, M., Escartí, A. y Pascual, C. (2011). Relaciones entre empatía, conducta prosocial, agresividad, autoeficacia y responsabilidad personal y social de los escolares, Psicothema, 23(1), 13-19.

Hemphill, J. (2003). Interpreting the magnitudes of correlation coefficients. American Psychologist, 58(1), 78-80. https://doi. org/10.1037/0003-066x.58.1.78

Jerusalem, M. \& Schwarzer, R. (1992). Self-eficacy as a resource factor in stress appraisal processes. En R. Schwarzer (Ed.), Self-efficacy: Thought control of action (pp. 195-213). Washington, DC: Hemisphere.

Luszczynska, A., Gibbons, F. X., Piko, B. \& Tekozel, M. (2004). Self-regulatory cognitions, social comparison, perceived peers' behaviors as predictors of nutrition and physical activity: 
A comparison among adolescents in Hungary, Poland, Turkey, and USA. Psychology and Health, 19, 577-593. https://doi.org/ 10.1080/0887044042000205844

Luszczynska, A., Scholz, U. \& Schwarzer, R. (2005). The General SelfEfficacy Scale: Multicultural validation studies, The Journal of Psychology, 139(5), 439-457.https://doi.org/10.3200/jrlp.139.5. 439-457

Martínez Arias, R. (1996). Psicometría: teoría de los test psicológicos y educativos. Madrid: Síntesis.

Morata-Ramírez, M. A. \& Holgado-Tello, F. T. (2013). Construct validity of Likert scales through confirmatory factor analysis: A simulation study comparing different methods of estimation based on Pearson and Polychoric correlations. International Journal of Social Sciences Studies, 1(1), 54-61. https://doi.org/ 10.11114/ijsss.v1i1.27

Ornelas, M., Blanco, H., Gastélum, G. y Chávez, A. (2012). Autoeficacia percibida en la conducta académica de estudiantes universitarias. Formación universitaria, 5(2), 17-26. http://dx. doi.org/10.4067/S0718-50062012000200003

Peinado, J. E., Ornelas, M., Blanco, J.R. y González, M.A. (2015). Invarianza Factorial de la Escala de Autoeficacia en la Solución de Problemas y Comunicación en Estudiantes Universitarios. Formación Universitaria, 8(4), 85-92. http://dx.doi.org/10.4067/ S0718-50062015000400010

Prieto L. (2001). La autoeficacia en el contexto académico: exploración bibliográfica comentada. Miscelánea Comillas, 59, 281-292.

Qualter, P., Dacre Pool, L., Gardner, K. J., Ashley-Kot, S., Wise, A. \& Wols, A. (2015). The Emotional Self-Efficacy Scale: Adaptation and validation for young adolescents. Journal of Psychoeducational Assessment, 33(1), 33-45. http://dx.doi.org/ 10.1177/0734282914550383

Reigal Garrido, R. E., Videra García, A., Martín Tamayo, I. y Juárez Ruiz de Mier, R. (2013). Importancia del autoconcepto físico y la autoeficacia general en la predicción de la conducta de práctica 
física. Apunts. Educación Física y Deportes, 112, 46-51. http:// dx.doi.org/10.5672/apunts.2014-0983.es.(2013/2).112.03

Rhemtulla, M., Brosseau, P. E. \& Savalei, V. (2012). When can categorical variables be treated as continuous? A comparison of robust continuous and categorical SEM estimation methods under suboptimal conditions. Psychological Methods, 17(3), 354373. https://doi.org/10.1037/a0029315

Rivera Heredia, M., Martínez Fuentes, M.,González Betanzos, F. y Salazar García, M. (2016). Autoeficacia, participación social y percepción de los servicios universitarios según el sexo. Revista de Psicología, 25(2), 1-16.

Salanova, M., Grau, R., Llorens, S. y Schaufeli, W.B. (2001). Exposición a las tecnologías de la información, burnout y engagement: el rol modulador de la autoeficacia relacionada con la tecnología. Revista de Psicología Social Aplicada, 11, 69-90.

Sansinenea, E., Gil de Montes, L., Agirrezabal, A., Larrańaga, M., Ortiz, G., Valencia, J. F. y Fuster, M. J. (2008). Autoconcordancia y autoeficacia en los objetivos personales: ¿Cuál es su aportación al bienestar? Anales de Psicología, 24(1), 121-128.

Smith, R.E. (1989). Effects of coping skills training on generalized self-efficacy and locus of control. Journal of Personality and Social Psychology, 56(2), 228-233. http://dx.doi.org/10.1037/ 0022-3514.56.2.228

Tinsley, H. E. \& Weiss, D. J. (1975). Interrater reliability and agreement of subjective judgments. Journal of Counseling Psychology, 22(4), 358-376. https://doi.org/10.1037/h0076640

Recibido: 9 de abril, 2018 Revisado: 28 de setiembre, 2018 Aceptado: 22 de febrero, 2019 